Article

\title{
An Integrated Study of Toxocara Infection in Honduran Children: Human Seroepidemiology and Environmental Contamination in a Coastal Community
}

\author{
Sergio A. Hernández ${ }^{1}$, José A. Gabrie ${ }^{1}{ }^{\circledR}$, Carol Anahelka Rodríguez ${ }^{2}\left({ }^{\circ}\right.$, Gabriela Matamoros $^{1,2}$, \\ María Mercedes Rueda ${ }^{2}$, Maritza Canales ${ }^{2}$, Ronald Mergl ${ }^{3}$ and Ana Sanchez ${ }^{1,2, *}$ (i) \\ 1 Department of Health Sciences, Brock University, St. Catharines, ON L3S 2A1, Canada; \\ hernandez@brocku.ca (S.A.H.); jgabrie@brocku.ca (J.A.G.); gm18@brocku.ca (G.M.) \\ 2 Department of Parasitology, School of Microbiology and Institute of Microbiology Research, \\ National Autonomous University of Honduras, Tegucigalpa, Honduras; \\ carol.rodriguez@unah.edu.hn (C.A.R.); maria.rueda@unah.edu.hn (M.M.R.); \\ maritza.canales@unah.edu.hn (M.C.) \\ 3 Niagara Falls Animal Medical Centre, Niagara Falls, ON L2E 6Z8, Canada; mergl.ron@gmail.com \\ * Correspondence: ana.sanchez@brocku.ca
}

Received: 22 June 2020; Accepted: 20 August 2020; Published: 23 August 2020

check for updates

\begin{abstract}
Background: Infections caused by Toxocara canis and T. cati are considered zoonoses of global importance. Reports from North and South America indicate that human infections are widespread in both continents, but epidemiological information from Central America is still lacking. (2) Methodology: In the present cross-sectional multi-year study, we aimed to undertake the first seroepidemiological and environmental study on toxocariasis in Honduras. This included the determination of seroprevalence of anti-Toxocara spp. antibodies in children using a Toxocara spp. purified excretory-secretory antigens enzyme-linked immunosorbent assay (TES-ELISA) and a confirmatory Western blot. As well, through statistical analysis including logistic regression we aimed at identifying relevant biological and epidemiological factors associated with seropositivity. The study also entailed detection of parasites' eggs in the soil samples both through Sheather's concentration method and a nested polymerase chain reaction-restriction fragment length polymorphism (PCR-RFLP) method. (3) Results: The study was undertaken in a coastal community of Honduras in 2 different years, 2015 and 2017. A total of 88 healthy schoolchildren completed the study, with participation of 79\% (73/92) and 65\% (46/71) of the student body in 2015 and 2017, respectively. Thirty-one children participated in both years (i.e., dual participants). Through both serological tests, seropositivity was confirmed in $88.6 \%$ (78/88) of children. Due to the high number of seropositives, logistic regression analysis was not possible for most socio-economic and epidemiological variables. Eosinophilia, on the other hand, was associated with seropositivity, independently of other intestinal helminthic infections. Continued seropositivity was observed in most of the dual participants, while seroconversion was determined in 8 of these children. Microscopic examination of soil samples did not yield any positive results. Through nested PCR-RFLP, 3 of the 50 samples $(6 \%)$ were positive for Toxocara spp.; two were identified as T. canis and one as T. cati. (4) Conclusions: This work documents for the first time, high levels of human exposure to Toxocara spp. in Honduras. These findings, along with the country's favorable epidemiological conditions for this zoonosis, emphasize the need for more research to determine whether this infection is underreported in the country.
\end{abstract}

Keywords: Toxocara; toxocariasis; zoonosis; seroepidemiology; neglected tropical diseases; Honduras 


\section{Introduction}

Toxocara spp. are cosmopolitan zoonotic parasites that utilize dogs, cats, foxes and other canids and felids as definitive hosts. When harboring adult worms in their intestine, these animals extensively contaminate their surroundings with their stools containing parasite ova [1]. Toxocara species are distributed worldwide, with higher prevalence where infected domestic dogs and cats are allowed to defecate in public spaces [2]. Once fully developed in the environment, Toxocara eggs are infectious to definitive hosts as well as to humans. In the latter, however, the parasites do not reach adult stages but rather lodge in tissues as larval stages causing a wide spectrum of pathologies grouped under the clinical term toxocariasis (also called 'toxocarosis') [3].

The significance of human toxocariasis as a disease remains enigmatic, partly due to the multifaceted, nonspecific and cryptic nature of symptoms, making this an insidious disease more closely related to disability and infirmity than mortality. Further, toxocariasis can lead to significant and irreversible damage such as blindness and fibrotic lesions in visceral organs. Recent research suggests that this infection may partially account for cognitive deficits and other neurological complications seen among socioeconomically disadvantaged children [1,4]. There is a strong body of research from Europe and South America and a recent interest resurgence in the United States [5,6].

Conversely, the epidemiological situation of toxocariasis in Central America is largely unknown $[7,8]$. Even in Latin America and the Caribbean (LAC) nations, where other neglected tropical diseases (NTDs) are well-characterized, toxocariasis has not been consistently studied and no estimates of regional prevalence have been calculated [8]. Despite that data show that Toxocara is an important infection in dogs and probably in cats in Central America [9], a recent review by Ma et al. brings to light the paucity of research on human Toxocara infection in this particular geographic region [10].

Among Central American countries, Honduras is a country that, due to its climatic and socio-economic characteristics, is endemic for several NTDs and other infections [8]. With over $60 \%$ of the population living in poverty (i.e., earning $<\$ 2$ USD/day) [11-13], and with a large uncontrolled population of domestic cats and dogs, the country offers optimal conditions for Toxocara spp. transmission; yet data on toxocariasis is almost non-existent $[4,7,8]$.

In the present study, we aimed to undertake the first seroepidemiological and environmental study on toxocariasis in Honduras. Firstly, we set out to determine the seroprevalence of anti-Toxocara spp. antibodies in children as an indicator of exposure to the parasite. Secondly, we sought out to investigate potential associations between seroprevalence and relevant biological and epidemiological factors. Finally, we conducted an environmental sampling to confirm that soil in public spaces could be one source of infection for the study population.

\section{Materials and Methods}

\subsection{Study Design and Population}

The present investigation was designed as an exploratory, cross-sectional study. A non-probability, purposive sampling method (based on expert knowledge of the population) was used to obtain the study sample. A minimum sample size was not calculated. Rather, research participants were recruited from a primary school population with high prevalence of soil-transmitted helminth (STH) infections. Two data collection visits took place: in August 2015 and October 2017.

The study was conducted in the village of Santa Cruz del Junco, within the municipality of Tela, department of Atlántida, on the northern Caribbean coast of Honduras.

The municipality has an area of $1196 \mathrm{~km}^{2}$ and an elevation of $3 \mathrm{~m}$ above sea level. The most recent census (2013) by the Honduran National Institute of Statistics recorded a population of 96,758 inhabitants, of which $48.8 \%$ were rural residents and $32.8 \%$ were $\leq 14$ years old. The same census also documented a poverty index of $51 \%$, with $42 \%$ of Tela's inhabitants listing agriculture, animal husbandry or fishing as their main source of income [14]. According to the Köppen Climate Classification, Tela exhibits a tropical rainforest climate, characterized by a lack of dry or wet seasons, 
as all months present at least $60 \mathrm{~mm}$ of precipitation; a condition that contributes to high levels of humidity. There is also no defined summer or winter in Tela, and it is typically hot and wet year-round [15].

The city of Tela, named after the municipality, is located between $15^{\circ} 47^{\prime} 00^{\prime \prime}$ North latitude and $87^{\circ} 2800^{\prime \prime}$ West longitude, placing it approximately $67 \mathrm{~km}$ north-east of the city of San Pedro Sula, the primary industrial center in Honduras and the nation's second largest city after the capital [14]. The study village is located approximately $11 \mathrm{~km}$ from the city's center.

A national survey investigating prevalence and intensities of soil-transmitted helminths (STH) in Honduran schoolchildren had identified Tela as an area with an STH prevalence close to $50 \%$ [12]. These conditions are ideal for the transmission of a variety of parasites, including Toxocara spp. [16,17].

\subsection{Study Sample}

Schoolchildren from the village's only public primary school (consisting of grades one through six), were invited to participate in the study, with no exclusion criteria. We focused on school-age children to determine exposure to Toxocara spp. based on their known contact with soil and propensity to harbor STH more than adults [8]. It is widely known that Toxocara infection is markedly more associated with children, a finding related to certain determinants such as geophagia, playing habits and hygienic practices [18-20].

\subsection{Data Collection}

Each participant's parent/guardian partook in a face-to-face interview that used a pre-developed questionnaire as a guideline for data collection. The questionnaire was structured into different categories aiming to gather information including demographic and epidemiological data, domestic animal presence in the household, relevant patterns of child behavior and knowledge of parasites.

\subsection{Blood Sample Collection and Analysis}

Sera collected from each participating child was tested for the detection of anti-Toxocara IgG antibodies by way of a commercially available ELISA kit (Diagnostic Automation, Inc./Cortez Diagnostics, Calabasas, CA, USA). According to manufacturer instructions, any sample that yielded an absorbance $\geq 0.3$ optical density (OD) units was considered seropositive. All positive ELISA results were confirmed by a Western blot (WB) assay, as well as 3 out of the 8 ELISA-negative samples. As per manufacturer instructions, the presence of two or more bands of low molecular weight were regarded as a positive result (LDBIO Diagnostics, Lyon, France). All assays were conducted according to manufacturer instructions. During the 2015 phase of the study, an eosinophil count was performed by an independent laboratory (Hospital CEMESA clinical laboratory), located in San Pedro Sula city, 1 h-drive from the study site. Eosinophilia was defined as $\geq 500$ eosinophils/ $\mu \mathrm{L}$ [21].

\subsection{Stool Sample Collection and Analysis}

In order to rule out potential serum cross-reactivity due to Ascaris lumbricoides infection as well as other parasitic infections characterized by eosinophilia, we analyzed children's stool samples for STH. A single stool sample was collected from each child and analyzed the same day at the local hospital laboratory using the Kato-Katz technique (Vestergaard Frandsen, Lausanne, Switzerland). With a Kato-Katz template delivering $41.7 \mathrm{mg}$ of sample, we used a factor of 24 to obtain the number of eggs per gram (epg) of stool. Kato-Katz smears were microscopically examined after $30 \mathrm{~min}$ of clarifying time. Any insufficient or unsuitable (not formed) stool sample was analyzed by direct wet mount examination. 


\subsection{Soil Sample Collection and Analysis}

A total of 50 soil samples, each approximately weighing $30 \mathrm{~g}$, were collected from 5 different sites in the community of Santa Cruz del Junco in 2017 (10 samples per site). Samples were collected from the superficial layer of soil, up to a depth of $5 \mathrm{~cm}$, avoiding any pebbles or grass. Soil samples were exported according to international regulations to Brock University in Canada for further microscopic and molecular analysis (permit number P-2018-00845).

Each sample was dried overnight at $37^{\circ} \mathrm{C}$, sieved with a $150 \mu \mathrm{m}$ pore size sifter and the resulting refined soil was kept at room temperature in $50 \mathrm{~mL}$ conical tubes. Volumes obtained through this process varied from 3 to $13 \mathrm{~mL}$.

The detection of Toxocara spp. in the refined samples, was done through a centrifugation/passive flotation technique (i.e., Sheather's) and a nested PCR-RFLP. For the Sheather's technique, samples were analyzed in duplicates as follows: $1 \mathrm{~g}$ of soil was transferred to a $15 \mathrm{~mL}$ centrifuge tube, mixed with $9 \mathrm{~mL}$ of distilled water, vortexed for one minute and then centrifuged at $1500 \times g$ for $5 \mathrm{~min}$. The resulting supernatant was discarded, and a flotation sucrose solution (Specific Gravity, SG 1.27) was added to the pellet to complete a volume of $10 \mathrm{~mL}$. Tubes were vortexed and filled with the same sucrose solution until a positive meniscus formed. Cover slips were placed on top of the tubes and, after a 60 -min period of passive flotation, were examined microscopically at $10 \times$ and $40 \times$ magnifications for the presence of Toxocara spp. eggs.

For the molecular detection of Toxocara spp., DNA was extracted from a $250 \mathrm{mg}$ aliquot of the refined soil using a commercial kit (Norgen Soil DNA Isolation Plus Kit cat\# 64040, Norgen Biotek Corp., Thorold, ON, Canada). Extraction was performed according to the manufacturer's protocol, with two modifications: (i) a preparatory step consisting of thermal stress, and (ii) a substitution of the beads provided with the kit. The thermal stress procedure entailed three rounds of a freeze-heat cycle, whereby samples were placed at $-80{ }^{\circ} \mathrm{C}$ for $10 \mathrm{~min}$ followed by dry incubation at $90{ }^{\circ} \mathrm{C}$ for 10 additional minutes. This thermal stress step was followed by overnight incubation with proteinase $\mathrm{K}$ at $56^{\circ} \mathrm{C}$. For the second modification, the plastic beads in each bead tube were replaced with a medley of sterile stainless-steel beads measuring $3 \mathrm{~mm}, 2 \mathrm{~mm}$ and $1.5 \mathrm{~mm}$.

A nested PCR approach, targeting segments of the 18S rRNA gene, was performed using the following primers: PCR 1: NC5f $5^{\prime}$-GTAGGTGAACCTGCGGAAGGATCATT- $3^{\prime}$ and NC2r 5'-TTAGTTTCTTTTCCTCCGCT-3' and PCR 2: FM1f 5'-TTGAGGGGAAATGGGTGAC-3' and FM2r $5^{\prime}$-TGCTGGAGGCCATATCGT-3'. Each PCR mix contained $10 \mu \mathrm{M}$ for both forward and reverse primers and $2 \mu \mathrm{L}$ of template DNA, with a final volume of $25 \mu \mathrm{L}$. The cycling conditions for PCR 1 were as follows: $94{ }^{\circ} \mathrm{C}$ for $3 \mathrm{~min}$; followed by 35 cycles of $94{ }^{\circ} \mathrm{C}$ for $45 \mathrm{~s}, 60^{\circ} \mathrm{C}$ for $1 \mathrm{~min}, 72{ }^{\circ} \mathrm{C}$ for $1 \mathrm{~min}$ and a final extension of $72{ }^{\circ} \mathrm{C}$ for $6 \mathrm{~min}$. For PCR 2, the cycle involved $94{ }^{\circ} \mathrm{C}$ for $3 \mathrm{~min} ; 35$ cycles of $94{ }^{\circ} \mathrm{C}$ for $30 \mathrm{~s}, 60^{\circ} \mathrm{C}$ for $30 \mathrm{~s}$ and $72{ }^{\circ} \mathrm{C}$ for $1 \mathrm{~min}$ and a final extension of $72{ }^{\circ} \mathrm{C}$ for $5 \mathrm{~min}$. The PCR was optimized for the detection of DNA originating from a single egg using spiked soil samples as well as soil negative controls.

Two restriction enzymes, Sall and Mval, were used to differentiate between T. canis and T. cati [13]. For this, amplicons were digested at $37^{\circ} \mathrm{C}$ for $3 \mathrm{~h}$. Digestion of the product by Sall $1500 \mathrm{U}$ (ThermoScientific ER0641, ThermoFisher Scientific, Waltham, MA, USA) produced 2 fragments of $\sim 320 \mathrm{bp}$ and $\sim 394 \mathrm{bp}$ in T. canis and an undigested band of $736 \mathrm{bp}$ for T. cati. While digestion by Mval (BstNI) $2000 \mathrm{U}$ (ThermoScientific ER0551, ThermoFisher Scientific) yielded 2 fragments of $\sim 600 \mathrm{bp}$ and $\sim 100 \mathrm{bp}$ in T. cati and an undigested band of $\sim 700 \mathrm{bp}$ for T. canis. Both PCR products and digested fragments were separated in $1.5 \%$ agarose gels with ethidium bromide.

\subsection{Statistical Analyses}

Data were inputted into a Microsoft Excel 2016 spreadsheet (Microsoft Corp., Redmond, WA, USA), cleaned for errors or missing values and exported for analysis to the STATA 13 software package (StataCorp LP., College Station, TX, USA). 
For characterization of the study population, we used descriptive statistics for both continuous and categorical variables. Seroprevalence of anti-Toxocara antibodies was calculated with the confirmatory test. Assessment of the significance of univariate associations with positive serology (as confirmed by WB) was done by Fisher's exact test. A logistic regression model was applied to investigate the relationship between the various epidemiological risk factors with anti-Toxocara antibody presence. Odds ratios (OR) were determined with $95 \%$ confidence intervals (CI).

Sensitivity, specificity, positive predictive, and negative predictive values were calculated for the ELISA, compared to the Western blot. The kappa statistic for level of agreement between both diagnostic tools was also evaluated. The level of agreement was interpreted as moderate, strong or almost perfect if the value of kappa was $0.60-0.79,0.80-0.90$ or $>0.90$, respectively [22]. For the eosinophilia measured in the first phase of the study, a Mann-Whitney $U$ test was employed to determine if there was a significant difference in circulating eosinophil counts in seropositive vs. seronegative individuals. Level of significance was established at $\alpha=0.05$.

\subsection{Ethics Statement}

Both phases of the present study received ethics clearance from both participating institutions, in Canada (Brock University; file number: 17-032-Sanchez, clearance received 12 September 2017; file number: 14-224-Sanchez, clearance received: 8 May 2015) and Honduras (National Autonomous University of Honduras, Tegucigalpa M.D.C, Honduras. Committee of Ethics, Master's program in Zoonotic and Infectious Diseases School of Microbiology; file number: 04-2017, clearance received: 21 September 2017; file number: 01-2015, clearance received: 1 August 2015). In addition, approval for the implementation of this study was requested from the participating school's principal and grade teachers. Both parental consent and children's assent were also required prior to an individual's participation.

\section{Results}

\subsection{Study Participation and Characterization of the Study Population}

A total of 88 different children completed the study. Enrolments were 73 and 46 for 2015 and 2017, respectively, but there were 31 children coincidentally enrolled in both years. These participants were deemed "dual participants". Study participation varied between years: in 2015, participation was 79\% of the school enrolment (73/92) whereas for 2017 it was only 65\% (46/71). The reason for the latter was a widespread outbreak of hemorrhagic conjunctivitis in Honduras (and throughout Latin America and the Caribbean) due to coxsackievirus A24, which prevented children's attendance to school and their potential enrolment in the study.

The final study sample was comprised of students between the ages of 6-15 years (mean 9.83 years \pm 2.25 ) and $45(51.1 \%)$ girls. A summary of the epidemiological and serological data and children's behavior is displayed in Table 1. An important proportion (71.6\%) of interviewed participants reported dog ownership by their family (Table 1). Additionally, most participants with household pets, either dogs or cats, specified that these animals strayed from the household freely ( $72.6 \%$ and $66.7 \%$, respectively). Among the behaviors listed, it is important to mention that $80.4 \%$ of children said they had some sort of contact with soil, mostly with soil from the playground adjacent to the school.

\subsection{Seroprevalence of Anti-Toxocara Antibodies}

Of the 88 serum samples, 80 were ELISA-positive. Western blot results (WB) confirmed that 78 out of the 80 sera were indeed positive for anti-Toxocara antibodies and hence, an overall seroprevalence of $88.6 \%$ was documented (Table 1). As a manner of internal quality control, we tested with WB 3 out of the $8(37.5 \%)$ ELISA-negative samples. The WB confirmed the absence of specific antibodies in these 3 samples. This was in addition to including the kit's negative controls. 
Table 1. Study sample characteristics and laboratory results by year of study. Since there were 31 children who participated in both years, the final sample size amounts to a total of $n=88$.

\begin{tabular}{|c|c|c|c|}
\hline Characteristics & $\begin{array}{c}2015 \\
n=73(\%)\end{array}$ & $\begin{array}{c}2017 \\
n=46(\%)\end{array}$ & $\begin{array}{c}\text { Total } \\
n=88(\%)\end{array}$ \\
\hline Males & $35(48 \%)$ & $24(52.2 \%)$ & $43(48.9 \%)$ \\
\hline Females & $38(52 \%)$ & $22(47.8 \%)$ & $45(51.1 \%)$ \\
\hline Age (years), mean $(S D)$ & $10.3(1.98)$ & $9.40(2.25)$ & $9.83(2.25)$ \\
\hline \multicolumn{4}{|c|}{ Soil Transmitted Helminthiases (STH) Profile } \\
\hline Overall STH prevalence & $30(41.1 \%)$ & $10(21.7 \%)$ 㧊 & $34(38.6 \%)$ 护 \\
\hline Ascaris lumbricoides infection & $10(13.7 \%)$ & $2(4.3 \%)$ 抽 & $10(11.4 \%)$ 怙 \\
\hline Trichuris trichiura infection & $26(35.6 \%)$ & $10(21.7 \%)$ 㧊 & 30 (34.1\%) 拉 \\
\hline Hookworm infection & $6(8.2 \%)$ & $0(0 \%)$ 抽 & $6(6.8 \%)$ 抽 \\
\hline Polyparasitic infections & $13(17.8 \%)$ & $2(4.3 \%)$ & $11(12.5 \%)$ \\
\hline Awareness of STH & $57(78.1 \%)$ & $24(52.2 \%)$ & $62(70.5 \%)$ \\
\hline Recalled having STH infection & $48(65.7 \%)$ & $24(52.2 \%)^{\S}$ & $54(71.1 \%) \S$ \\
\hline \multicolumn{4}{|c|}{ Anti-Toxocara Antibody Serology and Eosinophilia } \\
\hline Positive by TES-ELISA & $56(76.7 \%)$ & $45(97.8 \%)$ & $80(90.9 \%)$ \\
\hline Confirmed by Western Blot & $56(100 \%)$ & $43(95.6 \%)$ & $78(97.5 \%)$ \\
\hline Eosinophilia ( $\geq 500$ eosinophils $/ \mu \mathrm{L}) *$ & $16(21.9 \%)$ & NA & $16(21.9 \%) *$ \\
\hline \multicolumn{4}{|c|}{ Domestic Animal Conditions } \\
\hline Dog ownership $(n=88)$ & $53(72.6 \%)$ & $34(73.9 \%)$ & $63(71.6 \%)$ \\
\hline Free-ranging owned dogs & $39(73.5 \%)$ & $23(67.6 \%)^{+}$ & $45(72.6 \%)^{+}$ \\
\hline Cat ownership $(n=46)$ & NA & $24(52.1 \%)^{\dagger}$ & $24(52.1 \%)^{\dagger}$ \\
\hline Free-ranging owned cats & NA & $16(66.7 \%)^{\dagger}$ & $16(66.7 \%)^{\dagger}$ \\
\hline \multicolumn{4}{|c|}{ Children's Behavior $^{+}$} \\
\hline Contact with soil in the village & NA & $37(80.4 \%)$ & $37(80.4 \%)$ \\
\hline Contact with soil in school playground & NA & $41(91.1 \%) \lll \lll$ & $41(91.1 \%) \lll$ \\
\hline Geophagia & NA & $2(4.3 \%)$ & $2(4.3 \%)$ \\
\hline Onychophagia & NA & $11(23.9 \%)$ & $11(23.9 \%)$ \\
\hline Thumb-sucking & NA & $4(8.7 \%)$ & $4(8.7 \%)$ \\
\hline Consume undercooked beef & NA & $21(45.6 \%)$ & $21(45.6 \%)$ \\
\hline Consume raw fruits/vegetables & NA & $35(79.5 \%) \diamond \diamond$ & $35(79.5 \%) \diamond \diamond$ \\
\hline
\end{tabular}

NA: data not collected; ${ }^{*}$ data for 2015 only; ${ }^{\dagger}$ data collected for 2017 participants only; ${ }^{\ddagger}$ two children did not provide satisfactory stool samples for Kato-Katz examination; $\$$ data not recalled for 10 children; ${ }^{+}$data not recalled for one child; ${ }^{\diamond}$ data not recalled for two children; "' data not recalled for one child; ${ }^{\theta} 31$ dual participants counted only once. TES-ELISA: Toxocara spp. purified excretory-secretory antigens enzyme-linked immunosorbent assay

Of the seropositive participants, $52.6 \%$ (41/78) were males, but neither the univariate analysis nor the logistic regression model identified male sex as statistically significant for seropositivity $(p=0.09$; $\mathrm{OR}=4.43,95 \% \mathrm{CI}=0.87-22.42, p=0.07)$. Due to the high number of children with positive serology (the primary outcome), logistic regression analysis was not possible for most variables. Table 2 shows the results of this analysis for the remaining variables none of which were identified as statistically significant. The results of the dual participants are demonstrated in Table 3. It can be seen that the proportion of seropositive children increased, as 8 of the 31 dual participants seroconverted in the two-year interim (Table 3). 
Table 2. Logistic regression analysis of Toxocara spp. seropositivity among the studied schoolchildren $(n=88)$.

\begin{tabular}{cccc}
\hline Variable & Odds Ratio (OR) & 95\% Confidence Interval (CI) & $p$ Value \\
\hline Gender (Male) & 4.43 & $0.87-22.42$ & 0.072 \\
Soil Contact & 2.19 & $0.17-27.96$ & 0.547 \\
Onychophagia & 0.61 & $0.04-7.61$ & 0.698 \\
Raw Beef Consumption & - & - & - \\
Raw Fruit/Vegetable Consumption & 4.25 & $0.23-78.01$ & 0.330 \\
Dog Ownership & 1.80 & $0.46-7.10$ & 0.396 \\
Dog Age $\leq$ Year & - & - & - \\
Playground Contact with Soil & - & - & - \\
\hline
\end{tabular}

(-): Variable omitted from final model.

Table 3. Serological status of "dual participants": schoolchildren who participated in both 2015 and 2017.

\begin{tabular}{cccccc}
\hline Participants & $\begin{array}{c}\text { ELISA Positives } \\
\mathbf{2 0 1 5} \boldsymbol{n} \mathbf{( \% )}\end{array}$ & $\begin{array}{c}\text { Western Blot } \\
\text { Confirmed 2015 } \boldsymbol{n} \mathbf{( \% )}\end{array}$ & $\begin{array}{c}\text { ELISA Positives } \\
\mathbf{2 0 1 7} \boldsymbol{n} \mathbf{( \% )}\end{array}$ & $\begin{array}{c}\text { Western Blot } \\
\text { Confirmed 2017 } \boldsymbol{n} \mathbf{( \% )}\end{array}$ & $\begin{array}{c}\text { Seroconverted } \\
\text { by 2017 } \boldsymbol{n} \mathbf{( \% )}\end{array}$ \\
\hline Males $(n=15)$ & $12(80 \%)$ & $12(80 \%)$ & $15(100 \%)$ & $15(100 \%)$ & $3(20 \%)$ \\
Females $(n=16)$ & $9(56.3 \%)$ & $9(56.3 \%)$ & $15(93.7 \%)$ & $14(87.5 \%)$ & $5(31.3 \%)$ \\
Total $(n=31)$ & $21(67.7 \%)$ & $21(67.7 \%)$ & $30(96.7 \%)$ & $29(93.5 \%)$ & $8(26 \%)$ \\
\hline
\end{tabular}

\subsection{Toxocara spp. Seropositivity and Eosinophilia}

The eosinophil count performed in 2015 revealed eosinophilia (defined as $\geq 500$ eosinophils $/ \mu \mathrm{L}$ ) in 16 children out of the 73 participants (21.9\%). A Mann-Whitney $U$ test was done to see if there was a significant difference in the count of circulating eosinophils in schoolchildren who tested seropositive compared to those seronegative (Table 4). To account for possible confounders, the test was also applied when controlling for individuals with any kind of STH infection and T. trichiura specifically. A significant difference was found between both subgroups (Table 4). For those that were found free of any STH infection, Toxocara-seropositive children averaged 191.3 eosinophils $/ \mu \mathrm{L}$, compared to 101.2 eosinophils $/ \mu \mathrm{L}$ in those who were seronegative $(p=0.058)$. Increased eosinophil levels were also documented for those without trichuriasis $(p=0.035)$ (Table 4).

Table 4. Mann-Whitney $U$ test results comparing geometric mean of circulating eosinophil levels in seropositive vs. seronegative schoolchildren $(n=73)^{\dagger}$.

\begin{tabular}{cccc}
\hline Geometric Mean (G-Mean) Value & $\begin{array}{c}\text { Western Blot } \\
\text { Seropositives (95\% CI) }\end{array}$ & $\begin{array}{c}\text { Western Blot } \\
\text { Seronegatives (95\% CI) }\end{array}$ & $\boldsymbol{p}$ Value \\
\hline G-Mean * eosinophils $/ \mu \mathrm{L}$ & $262.1(211.7-324.4)$ & $101.2(59.6-171.9)$ & 0.004 \\
G-Mean eosinophils $/ \mu \mathrm{L}$ (without STH) & $191.3(141.7-258.2)$ & $101.2(59.6-171.9)$ & 0.058 \\
G-Mean eosinophils $/ \mu \mathrm{L}$ (without T. trichiura) & $198.5(151.2-260.5)$ & $101.2(59.6-171.9)$ & 0.035 \\
\hline
\end{tabular}

${ }^{\dagger}$ Eosinophilia data only collected in $2015 ;{ }^{*}$ G-Mean: geometric mean.

\subsection{Comparison of Serodiagnostic Techniques}

As an additional step, the performance of the TES-ELISA test used in this study was measured against the Western blot, the current recommended confirmatory test for anti-Toxocara antibody detection. The average ELISA sensitivity was 100\% (95\% CI 95.4-100\%) and the average specificity was $80 \%$ (95\% CI 44.4-97.5\%), lower than the $93.7 \%$ reported by the manufacturer. With these two parameters, a kappa $(\mathrm{K})$ statistic was calculated to establish the degree of agreement between the two serodiagnostic tests. In this case, the resulting kappa statistic was $K=0.87$, an indicator of a strong agreement between the two diagnostic tools. Additionally, the average ELISA's positive and negative predictive value (PPV) were 97.5\% (95\% CI: 91.3-99.7\%) and 100\% (95\% CI: 63.1-100\%), respectively. 


\subsection{Intestinal Parasitic Infections}

Two children provided insufficient or unsatisfactory stool samples for the Kato-Katz technique, so the samples were instead analyzed by direct wet mount. Overall, 34 of $88(38.6 \%)$ children were infected with at least one STH species of which T. trichiura was the most prevalent (30/88 or 34.1\%). Concurrent parasitoses were observed in 11 (12.5\%) participants, highlighting the possibility of potential cross-reacting antibodies (Table 1 ).

\subsection{Identification of Toxocara spp. in Soil Samples}

Microscopic examination after sucrose concentration of soil samples did not yield any positive results. However, eggs of T. trichiura and A. lumbricoides were detected with this method in $3(6 \%)$ samples.

In contrast, in the nested PCR-RFLP we identified 3 of the 50 samples (6\%) as positive for Toxocara spp.; two were identified as T. canis and one as T. cati DNA. These 3 samples were collected in two of the five collection sites, one of which turned out to be the playground adjacent to the school. These Toxocara positive samples were negative for any STH by microscopy after the Sheather's concentration technique.

\section{Discussion}

Adding to the list of neglected tropical diseases (NTDs) in Honduras, this work documents for the first time, high levels of human exposure to Toxocara spp. in the country, and suggests the potential for this infection to be seriously underreported.

To our knowledge, only one clinical case of toxocariasis has been published in Honduras. Puerto-Sanabria et al. [23] described in 2016 a case in a 14-month-old infant with central nervous system involvement. Canine toxocariasis is poorly documented as well, even though the infection is frequently treated at veterinary clinics (Sanchez A, personal observations). Reference to the infection circulating in Honduran puppies is made in a 2002 review paper by Javier and Alger [24]. In addition, through an exhaustive search of Latin American databases and Honduran journals, we were able to find two publications: a study published in the Honduran Medical Journal reporting a toxocariasis prevalence of $3.8 \%$ in a sample of 207 dogs ( 82 owned, 69 from a kennel, and 56 free-roaming) [25], and a conference poster presentation by Valle-Ramirez \& collaborators describing T. canis infection in $12 \%$ of 177 dogs examined [26].

Other than the publications mentioned above, we were not able to find more toxocariasis-related data either published or in the grey literature. Nonetheless, given that canine and human toxocariasis are prevalent in countries with similar climatic and socio-economic characteristics [6], we theorized that this parasitic disease is highly prevalent in Honduras.

The study findings confirmed our hypothesis —at least in the study sample — as we determined an overall seroprevalence of $88.6 \%$. This is a surprisingly high seropositivity, but with the use of a confirmatory test (i.e., Western blot), we are confident that our results are reliable. Such high seroprevalence warrants a clinical investigation as covert toxocariasis would be a serious concern among the studied children [3]. Further, evidence indicates strong links between seropositivity and cognitive and developmental delays $[1,4,5,27,28]$. To reinforce the plausible link between seropositivity and health effects, we found that after controlling for STH infections, the presence of anti-Toxocara antibodies was associated with high levels of eosinophilia. In fact, of 73 children tested in 2015, 16 had high counts of circulating eosinophils and all were seropositive. A difference was observed between the eosinophil geometric means of seropositive children when compared to those of seronegative ones (191.3 eosinophils/ $\mu \mathrm{L}$ vs. 101.2 eosinophils $/ \mu \mathrm{L}$, respectively), but this difference did not reach statistical significance $(p=0.058)$.

Many studies have already pointed out eosinophilia as a clinical marker for several helminthic and some protozoal infections [21]. Among helminthic infections, strongyloidiasis is commonly 
associated with strong eosinophilic reactions, whereas ascariasis and trichuriasis are known to be eosinophilia-inducing, albeit to a lesser degree [29-31]. A. lumbricoides, T. trichiura, and hookworm infections showed a pattern of endemic transmission among the studied children, with an overall prevalence of $38.6 \%$ across the 2-year period. The higher prevalence of trichuriasis is noteworthy-a fact that we have demonstrated in other Honduran communities and that had prompted us to investigate anthelminthic resistance in light of decades of deworming campaigns in the country [32].

In Honduras, strongyloidiasis is not reported frequently, especially in children [12]. Other parasitoses characterized by eosinophilia such as trichinellosis, filariasis, fascioliasis, echinococcosis, schistosomiasis, etc. [21,29,31-36] are not prevalent in the country.

Eosinophilia, on the other hand, has long been recognized as an important biomarker for toxocariasis [37-40]. In the major clinical presentation, visceral larva migrans (VLM) syndrome, eosinophilia is a distinguishable biomarker that can be drastically elevated [37,41]. In other clinical presentations for instance, ocular larva migrans (OLM), covert toxocariasis or neurotoxocariasis, eosinophilia can still be present, but on average, at lower levels than in VLM [20,37,42]. The concurrent findings of eosinophilia and seropositivity among the studied children are consistent with results from other studies [43-46]. Moreover, the combination of eosinophilia and seropositivity among the studied children may suggest the presence of an active infection (i.e., with viable larvae in tissues) [47]. A clinical and laboratory examination of these children could elucidate whether their seropositive status can be linked to covert (inapparent) or common toxocariasis [3].

Gender of the participating children was not identified as a statistically significant risk factor in our study either. It is interesting to note, however, that the proportion of seropositive boys (41/45, 95.35\%) was higher than that of girls $(37 / 45,82.22 \%)$, and that the logistic regression analysis identified boys at about 4-fold increased odds of having anti-Toxocara antibodies. It is generally speculated that this association is related to boys' increased exposure to infective eggs in the soil from outdoor activities as well as their proclivity to play and have more contact with animals than girls [48,49].

In terms of epidemiological factors associated to seropositivity, we aimed to identify those postulated in the literature (e.g., soil contact, geophagia, dog ownership, raw beef consumption) but unfortunately, the substantial levels of seropositivity within such a small sample prevented us from detecting statistically significant associations, notably between plausible variables such as soil contact, dog ownership, undercooked beef consumption, or geophagia.

In fact, with almost all children (95\%) reporting frequent exposure to soil, and almost $90 \%$ of them being seropositive, it would have been surprising to detect any statistical association between these two variables. Still, despite the lack of statistical significance, our logistic regression analysis detected that those reporting soil contact were twice as likely to have anti-Toxocara spp. antibodies.

We were also unable to identify the consumption of raw fruits or vegetables as a significant risk factor for anti-Toxocara spp. antibody presence. Yet, children who affirmed these dietary habits were found to be at four-times greater odds of being seropositive. A great proportion of children who admitted consuming raw fruits/vegetables were found to be seropositive $(34 / 35,97.14 \%)$.

Lastly, in terms of risk factors, even though the association was not significant, most children reporting family dog ownership (57/63) had almost twice the odds of being seropositive compared to their counterparts. This finding may reflect the considerable impact of these canine populations as a source of contamination, especially for children [50-52]. This is particularly true in areas such as the one studied here, where we observed free-ranging dogs running rampant with unrestricted access to public spaces. It would be important to conduct a canine seroepidemiological survey in the community - and in the country at large- to ascertain the size of the dog population and get an overview of the prevailing health issues including zoonotic pathogens such as toxocariasis.

A unique aspect of the present investigation is the testing of the study population in two different years, which allowed for data collection within the same community at two separate time points (2015 and 2017). Moreover, since the second phase of this study (2017) was carried out within the very same school as two years prior, overlap of some participants was inevitable. Initially, 21 of $31(67.7 \%)$ 
dual participants tested seropositive via TES-ELISA in 2015. In 2017, this proportion increased to 30 of the $31(96.7 \%)$. Of these 9 seroconverted participants, the confirmatory Western blot identified one as a false positive, leading to the conclusion that 8 children had been exposed to the parasite during the two-year interim. Seroconversion may be attributed to changes in social and recreational behaviors of children as they age. In the two-year study gap, these children could have experienced dietary changes or new/more frequent contact with definitive or paratenic hosts, contaminated environments or fomites, or acquisition or strengthening of unhygienic habits (e.g., geophagia, inadequate handwashing, etc.) $[2,18,53]$. Some authors suggest that seroconversion is not intrinsically related to behavior changes. They propose that an increase in antibody titers to detectable levels might be due to the cumulative effect of persistent exposure and infection, or to the constant antigenic stimulation elicited by live larvae in tissues [1]. While in the studied population all these scenarios are possible, the fact remains that there is in the community a continuous presence of infectious sources.

Finally, to further investigate potential sources of infection, we integrated into the study an environmental component. Out of the 50 soil samples collected from 5 community sites, 3 samples (6\%) from two sites contained Toxocara spp. eggs. Two of the three positive samples were collected from the playground adjacent to the school; an unfenced area that is heavily trafficked by children and animals, even outside school hours. In agreement with worldwide literature [54], our data suggest that the playground could be an important source of exposure to Toxocara spp. and if so, installing a fence around its perimeter would help mitigate the risk of exposure.

There are some limitations and strengths to this study. An important limitation that prevents generalization is the small sample size restricted to one cohort of schoolchildren. Not only did the study lack statistical power, but a cluster effect most certainly led to capturing a high prevalence phenomenon. Naturally, a small sample size and the extremely high seropositivity observed hindered meaningful statistical analyses. A second caveat worth mentioning pertains to the lack of clinical data from the studied children. Without investigating the potential health implications of a seropositive status, our study can only suggest that the high levels of exposure to Toxocara in this community underscore the need for appropriate attention from the health and veterinary sectors. One more limitation entails not including a canine survey. Although this was considered, we could not secure trained personnel to capture free-ranging dogs to obtain blood and stool samples.

Limitations notwithstanding, the study draws strength from the use of both an ELISA and a confirmatory Western blot, which lends reliability to our seroprevalence data. In addition, we were successful in detecting through PCR Toxocara spp. eggs in soil samples of 2 out of 5 collection sites. Further, we were able to identify both $T$. canis and $T$. cati in soil samples, opening research possibilities for the study of feline populations as well. A unique strength of the study is having a sub-sample of participants enrolled in both 2015 and 2017. This allowed us to run a nested comparison at two different time points and identify continued seropositivity as well as seroconversion.

In conclusion, we here present the first serological survey on human toxocariasis in Honduras, filling an important knowledge gap not only in the country but in the Central American region. Next steps should include conducting larger epidemiological, veterinary and clinical investigations using a One Health approach. Such data would inform an initial assessment of the burden of this neglected-almost invisible—zoonotic disease.

Author Contributions: Conceptualization, A.S., M.C. and R.M.; methodology, S.A.H., J.A.G., C.A.R., G.M., M.M.R., M.C. and A.S.; formal analysis, S.A.H., J.A.G., G.M., and A.S.; investigation, S.A.H.; J.A.G.; C.A.R.; G.M.; M.M.R.; M.C. and A.S.; resources, A.S., M.C., and R.M.; data curation, S.A.H., and J.A.G.; writing-original draft preparation, A.S., and S.A.H.; writing-review and editing, A.S., S.A.H., G.M., J.A.G. and R.M.; supervision, A.S. and M.C.; project administration, A.S. All authors have read and agreed to the published version of the manuscript.

Funding: This research received no external funding.

Acknowledgments: We are grateful to the municipal health authorities for their interest and support, and for making available Tela's Hospitals clinical laboratory for sample analyses. Our gratitude also goes to the school principal and teachers for their decisive support to the study. A special thanks to Sara Ávalos (Universidad Nacional 
Autónoma de Honduras) for her valuable participation in the field work, as well as to Anneliese von Eicken and Nick Orrego (Brock University) for assisting with laboratory work.

Conflicts of Interest: The authors declare no conflict of interest

\section{References}

1. Walsh, M.G.; Haseeb, M.A. Reduced cognitive function in children with toxocariasis in a nationally representative sample of the United States. Int. J. Parasitol. 2012, 42, 1159-1163. [CrossRef]

2. Chen, J.; Liu, Q.; Liu, G.H.; Zheng, W.B.; Hong, S.J.; Sugiyama, H.; Zhu, X.-Q.; Elsheikha, H.M. Toxocariasis: A silent threat with a progressive public health impact. Infect. Dis. Poverty 2018, 7, 59. [CrossRef]

3. Auer, H.; Walochnik, J. Toxocariasis and the clinical spectrum. Adv. Parasitol. 2020, 109, 111-130. [CrossRef] [PubMed]

4. Hotez, P.J. Neglected infections of Poverty in the United States and their effects on the brain. JAMA Psychiatry 2014, 71, 1099-1100. [CrossRef] [PubMed]

5. Tyungu, D.L.; McCormick, D.; Lau, C.L.; Chang, M.; Murphy, J.R.; Hotez, P.J.; Mejia, R.; Pollack, H. Toxocara species environmental contamination of public spaces in New York City. PLoS Negl. Trop. Dis. 2020, 14, e0008249. [CrossRef] [PubMed]

6. Rostami, A.; Riahi, S.M.; Holland, C.V.; Taghipour, A.; Khalili-Fomeshi, M.; Fakhri, Y.; Omrani, V.F.; Hotez, P.J.; Gasser, R.B. Seroprevalence estimates for toxocariasis in people worldwide: A systematic review and meta-analysis. PLoS Negl. Trop. Dis. 2019, 13, e0007809. [CrossRef]

7. Bolívar-Mejía, A.; Alarcón-Olave, C.; Calvo-Betancourt, L.S.; Paniz-Mondolfi, A.; Delgado, O.; Rodriguez-Morales, A.J. Toxocariasis in the Americas: Burden and disease control. Curr. Trop. Med. Rep. 2014, 1, 62-68. [CrossRef]

8. Hotez, P.J.; Bottazzi, M.E.; Franco-Paredes, C.; Ault, S.K.; Periago, M.R. The neglected tropical diseases of Latin America and the Caribbean: A review of disease burden and distribution and a roadmap for control and elimination. PLoS Negl. Trop. Dis. 2008, 2, e300. [CrossRef]

9. Ketzis, J.K.; Lucio-Forster, A. Toxocara canis and Toxocara cati in domestic dogs and cats in the United States, Mexico, Central America and the Caribbean: A review. Adv. Parasitol. 2020, 109, 655-714.

10. Ma, G.; Rostami, A.; Wang, T.; Hofmann, A.; Hotez, P.J.; Gasser, R.B. Global and regional seroprevalence estimates for human toxocariasis: A call for action. Adv. Parasitol. 2020, 109, 275-290. [CrossRef]

11. Sanchez, A.L.; Gabrie, J.A.; Usuanlele, M.T.; Rueda, M.M.; Canales, M.; Gyorkos, T.W. Soil-transmitted helminth infections and nutritional status in school-age children from rural communities in Honduras. PLoS Negl. Trop. Dis. 2013, 7, e2378. [CrossRef] [PubMed]

12. Torres, R.E.M.; Garcia, D.N.F.; Sandoval, G.A.F.; Santana, A.H.; Singh, P.; Bucheli, S.T.M.; Saboyá, M.; Paz, M.Y. Prevalence and intensity of soil-transmitted helminthiasis, prevalence of malaria and nutritional status of school going children in Honduras. PLoS Negl. Trop. Dis. 2014, 8, e3248. [CrossRef]

13. Mikaeili, F.; Mathis, A.; Deplazes, P.; Mirhendi, H.; Barazesh, A.; Ebrahimi, S.; Kia, E.B. Differentiation of Toxocara canis and Toxocara cati based on PCR-RFLP analyses of rDNA-ITS and mitochondrial cox1 and nad1 regions. Acta Parasitol. 2017, 62, 549-556. [CrossRef] [PubMed]

14. INE-Instituto Nacional de Estadísticas Honduras. Available online: https://www.ine.gob.hn/V3/ (accessed on 21 July 2020).

15. Climate and Average Monthly Weather in Tela, Honduras. Available online: https://weather-and-climate. com/average-monthly-Rainfall-Temperature-Sunshine-fahrenheit,Tela,Honduras (accessed on 21 July 2020).

16. Hotez, P.J.; Wilkins, P.P. Toxocariasis: America's most common neglected infection of poverty and a helminthiasis of global importance? PLoS Negl. Trop. Dis. 2009, 3, e400. [CrossRef]

17. Mizgajska, $\mathrm{H}$. The role of some environmental factors in the contamination of soil with Toxocara spp. and other geohelminth eggs. Parasitol. Int. 1997, 46, 67-72. [CrossRef]

18. Despommier, D. Toxocariasis: Clinical aspects, epidemiology, medical ecology, and molecular aspects. Clin. Microbiol. Rev. 2003, 16, 265-272. [CrossRef]

19. Macpherson, C.N. Human behaviour and the epidemiology of parasitic zoonoses. Int. J. Parasitol. 2005, 35, 1319-1331. [CrossRef]

20. Magnaval, J.F.; Glickman, L.T.; Dorchies, P.; Morassin, B. Highlights of human toxocariasis. Korean J. Parasitol. 2001, 39, 1-11. [CrossRef] 
21. Kovalszki, A.; Weller, P.F. Eosinophilia. Prim. Care. 2016, 43, 607-617. [CrossRef]

22. McHugh, M.L. Interrater reliability: The kappa statistic. Biochem. Med. (Zagreb). 2012, 22, 276-282. [CrossRef]

23. Puerto Sanabria, C.R.; Tovar, S. Infección al sistema nervioso por Toxocara canis en Hospital Escuela Universitario, Honduras. Rev. Fac. Cienc. Méd. 2016, 13, 47-51.

24. Javier, C.A.; Alger, J. Larva migrans visceral: Enfoque diagnóstico con énfasis en el inmunodiagnóstico. Rev. Med. Hond. 2002, 70, 125-126.

25. Kaminsky, R.; Groothousen, C.M.; Zúniga, A.M.; Contreras, M.; Ferrera, A.M.; Henríquez, K.C. Infección por Toxocara canis en perros y riesgo de toxocariasis humana, Honduras. Rev. Med. Hond. 2014, 82, 2-50.

26. Manuel, V.R.; Nelys, H.F.; David, C.P.; Saharai, D.M.Y.; Rubén, R.A. Prevalencia de Parasitos Intestinales en Caninos de la Ciudad de Catacamas; Departamento de Olancho: Juticalpa, Honduras, 2016; Volume 167.

27. Ma, G.; Holland, C.V.; Wang, T.; Hofmann, A.; Fan, C.-K.; Maizels, R.M.; Hotez, P.; Gasser, R.B. Human toxocariasis. Lancet Infect. Dis. 2018, 18, e14-e24. [CrossRef]

28. Holland, C.V.; Hamilton, C.M. The significance of cerebral toxocariasis: A model system for exploring the link between brain involvement, behaviour and the immune response. J. Exp. Biol. 2013, 216 Pt 1, 78-83. [CrossRef]

29. Mejia, R.; Nutman, T.B. Evaluation and differential diagnosis of marked, persistent eosinophilia. Semin. Hematol. 2012, 49, 149-159. [CrossRef]

30. Ustianowski, A.; Zumla, A. Eosinophilia in the returning traveler. Infect. Dis. Clin. N. Am. 2012, 26, 781-789. [CrossRef]

31. Salzer, H.J.; Rolling, T.; Vinnemeier, C.D.; Tannich, E.; Schmiedel, S.; Addo, M.M.; Cramer, J.P. Helminthic infections in returning travelers and migrants with eosinophilia: Diagnostic value of medical history, eosinophil count and IgE. Travel Med. Infect. Dis. 2017, 20, 49-55. [CrossRef]

32. Matamoros, G.; Rueda, M.M.; Rodríguez, C.; Gabrie, J.; Canales, M.; Fontecha, G.; Sánchez, A. High Endemicity of Soil-transmitted helminths in a population frequently exposed to albendazole but no evidence of antiparasitic resistance. Trop. Med. Infect. Dis. 2019, 4, 73. [CrossRef]

33. Taylor, M.J.; Hoerauf, A.; Bockarie, M. Lymphatic filariasis and onchocerciasis. Lancet 2010, 376, 1175-1185. [CrossRef]

34. Esteban, J.; Bargues, M.; Mas-Coma, S. Geographical distribution, diagnosis and treatment of human fascioliasis: A review. Res. Rev. Parasitol. 1998, 58, 13-42.

35. Brunetti, E.; Kern, P.; Vuitton, D.A. Writing Panel for the W-I. Expert consensus for the diagnosis and treatment of cystic and alveolar echinococcosis in humans. Acta Trop. 2010, 114, 1-16. [CrossRef] [PubMed]

36. Tang, Z.L.; Huang, Y.; Yu, X.B. Current status and perspectives of Clonorchis sinensis and clonorchiasis: Epidemiology, pathogenesis, omics, prevention and control. Infect. Dis. Poverty. 2016, 5, 71. [CrossRef] [PubMed]

37. Fillaux, J.; Magnaval, J.F. Laboratory diagnosis of human toxocariasis. Vet. Parasitol. 2013, 193, $327-336$. [CrossRef] [PubMed]

38. Pawlowski, Z. Toxocariasis in humans: Clinical expression and treatment dilemma. J. Helminthol. 2001, 75, 299-305. [CrossRef]

39. Yoon, S.Y.; Baek, S.; Park, S.Y.; Shin, B.; Kwon, H.S.; Cho, Y.S.; Moon, H.-B.; Kim, T.-B. Clinical course and treatment outcomes of toxocariasis-related eosinophilic disorder. Medicine (Baltimore) 2018, 97, e12361. [CrossRef]

40. Kwon, N.-H.; Oh, M.-J.; Lee, S.P.; Lee, B.-J.; Choi, D.-C. The prevalence and diagnostic value of toxocariasis in unknown eosinophilia. Ann. Hematol. 2006, 85, 233-238. [CrossRef]

41. Lassmann, B.; Tsigrelis, C.; Virk, A. 33-year-old woman with marked eosinophilia. Mayo Clin. Proc. 2007, 82, 103-106. [CrossRef]

42. Ikotun, K.; Sowemimo, O.; Chou, C.-M.; Ajenifuja, K.; Chuang, T.-W.; Asaolu, S.; Akinwale, O.; Gyang, V.; Nwafor, T.; Henry, E.; et al. High seroprevalence of Toxocara antibodies in pregnant women attending an antenatal clinic at a university hospital in Ile-Ife, Nigeria. Trans. R. Soc. Trop. Med. Hyg. 2020, 114, 301-307. [CrossRef]

43. Ciarmela, M.L.; Pezzani, B.C.; Minvielle, M.C. Toxocariasis, intestinal parasitoses and eosinophilia in schoolchildren from Argentina. Curr. Health Sci. J. 2016, 42, 5-11. [CrossRef] 
44. Roldan, W.H.; Espinoza, Y.A.; Huapaya, P.E.; Huiza, A.F.; Sevilla, C.R.; Jimenez, S. Frequency of human toxocariasis in a rural population from Cajamarca, Perú determined by DOT-ELISA test. Rev. Inst. Med. Trop. Sao Paulo. 2009, 51, 67-71. [CrossRef] [PubMed]

45. Martínez, M.; Garcia, H.; Figuera, L.; Gonzalez, V.; Lamas, F.; López, K.; Mijares, V.; Corrales, Y.; Lares, M.; Ferrer, E. Seroprevalence and risk factors of toxocariasis in preschool children in Aragua state, Venezuela. Trans. R. Soc. Trop. Med. Hyg. 2015, 109, 579-588. [CrossRef]

46. Mendonça, L.R.; Veiga, R.V.; Dattoli, V.C.; Figueiredo, C.A.; Fiaccone, R.; Santos, J.; Cruz, A.A.; Rodrigues, L.C.; Cooper, P.J.; Pontes-De-Carvalho, L.C.; et al. Toxocara seropositivity, atopy and wheezing in children living in poor neighbourhoods in urban Latin America. PLoS Negl. Trop. Dis. 2012, 6, e1886. [CrossRef] [PubMed]

47. Łuzna-Lyskov, A.; Andrzejewska, I.; Lesicka, U.; Szewczyk-Kramska, B.; Luty, T.; Pawlowski, Z. Clinical interpretation of eosinophilia and ELISA values (OD) in toxocarosis. Acta Parasitol. 2000, 45, 35-39.

48. Roldan, W.; Espinoza, Y.; Atúncar, A.; Ortega, E.; Martinez, A.; Saravia, M. Frequency of eosinophilia and risk factors and their association with Toxocara infection in schoolchildren during a health survey in the North of Lima, Peru. Rev. Inst. Med. Trop. Sao Paulo. 2008, 50, 273-278. [CrossRef]

49. Holland, C.V.; O'Lorcain, P.; Taylor, M.R.; Kelly, A. Sero-epidemiology of toxocariasis in school children. Parasitology. 1995, 110 Pt 5, 535-545. [CrossRef]

50. Macpherson, C.N. The epidemiology and public health importance of toxocariasis: A zoonosis of global importance. Int. J. Parasitol. 2013, 43, 999-1008. [CrossRef]

51. Fan, C.K.; Liao, C.W.; Cheng, Y.C. Factors affecting disease manifestation of toxocarosis in humans: Genetics and environment. Vet. Parasitol. 2013, 193, 342-352. [CrossRef]

52. Overgaauw, P.A.; van Knapen, F. Veterinary and public health aspects of Toxocara spp. Vet. Parasitol. 2013, 193, 398-403. [CrossRef]

53. Correa, C.; Bismarck, C. Toxocariasis: Incidence, prevalence and the time serum remains positive in school children from Campinas, SP, Brazil. J. Trop. Pediatr. 2009, 56, 215-216. [CrossRef]

54. Fakhri, Y.; Gasser, R.; Rostami, A.; Fan, C.-K.; Ghasemi, S.; Javanian, M.; Bayani, M.; Armoon, B.; Moradi, B. Toxocara eggs in public places worldwide-A systematic review and meta-analysis. Environ. Pollut. 2018, 242 Pt B, 1467-1475. [CrossRef] 